\title{
News on the zbMATH Interface
}

Octavio Paniagua Taboada (FIZ Karlsruhe, Berlin, Germany)

Our staff of developers and editors have updated and improved several important features in zbMATH. All these updates aim to provide our users a rich and modern database interconnected with other worldwide databases and services (e.g. digital libraries, Wikidata, ORCID and links to discussions in MathOverflow).

\section{Reviewer service and compensation}

During the last few months, we have updated several components of the reviewer service and submission tool. It is now possible to check the account balance and order Springer books, as well as make donations to the EMS book programme for developing countries via the interface. From January 2018, the financial compensation per review will be increased to 3.00 EUR from the traditional amount of 2.56 EUR. When this amount is used to order Springer books, a discount of $50 \%$ is applied.

\section{zbMATH Remote}

zbMATH Remote (https://zbmath.org/remote_access/) provides access to zbMATH from your mobile device using your institution's subscription, even when you are not logged into the institution's network. This access is valid for a period of three months and can be renewed whenever logged into your institution's network. When connected to a licensing network, you can activate remote access to zbMATH by clicking on the "Remote" link located at the top right menu of the zbMATH website.

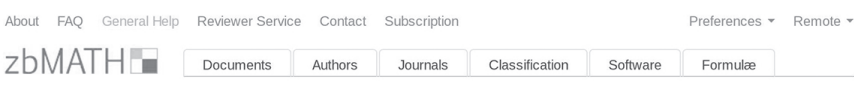

zbMATH Remote

This service allows you to access zbMATH for a period of 3 months from a mobile device using your institution's subscription. Please note that this service needs cookies to work. If you configured your browser to delete cookies when closing, you may want to grant an exception to

Remote Access License Agreement

By clicking the "Confirm" button below, I confirm that

HTH access by the license agreement(s) of the institution FI-Karlsruhe, which currenty

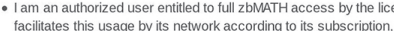

- I am solely responsible for use of my mobile device and should bear all consequences coming from misuse of my device.

- I accept the terms and conditions of this license.

In particular, lam aware tha

- this mobile access is limited in time and can only be extended by a renewed confirmation within an authorized license, and - any violation of these terms and conditions and/or the terms and conditions of the institution's license agreement(s) will result in the

Confim

\section{zbMATH interface features}

Search results can now be sorted using customised criteria such as publication year, document or review citation, and volume number.

Additional filters have been added. Formula search has been extended by the integration of arXiv full-texts, making more than 160 million mathematical formulas retrievable. A new interface available at MathOverflow allows users to insert a citation into any question, answer or comment, and enables linking back from zbMATH to a discussion there.
Here is an example of such a citation.

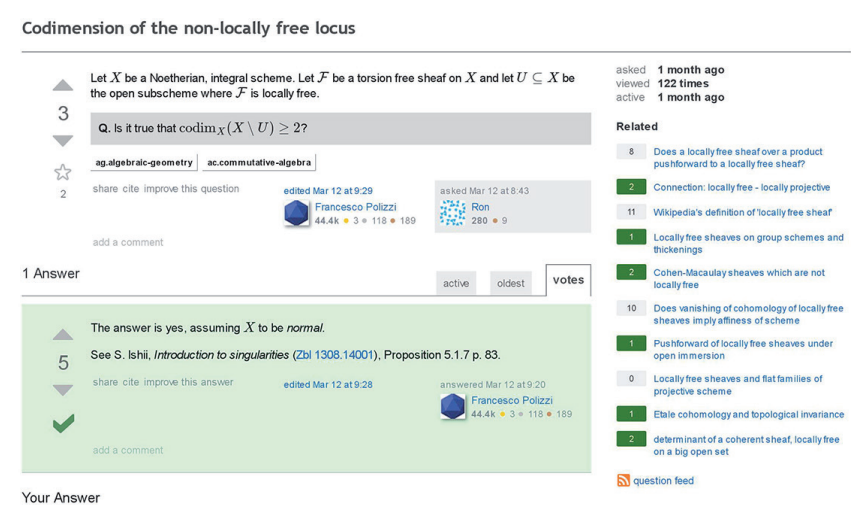

\section{MSC2020}

zbMATH and Mathematical Reviews have worked together to revise and improve the Mathematics Subject Classification (MSC) schema, which is used by these reviewing services and publishers to categorise items in mathematics literature. Comments and suggestions can be submitted through the website http://msc2020.org/.

\section{zbMATH Atom Feed}

zbMATH now offers an additional way of keeping researchers up-to-date with mathematical developments in their areas of interest via an atom feed. Every two weeks, researchers receive an update of recently included items and reviews in electronic form.

This feed is an enhanced version of the classic web feed format RSS and is supported by all current news readers.

If you are interested in a specific author, you can access this news feed through the author ID (displayed at the top of each author profile, e.g. https://zbmath.org/ atom/ai/leibniz.gottfried-wilhelm). You can also access the atom feed via MSC code, which can be defined as complex and specific as you wish (e.g. https://zbmath.org/ atom/cc/81,17B,57R56 for quantum theory, Lie algebras and Lie superalgebras, and topological quantum field theories).

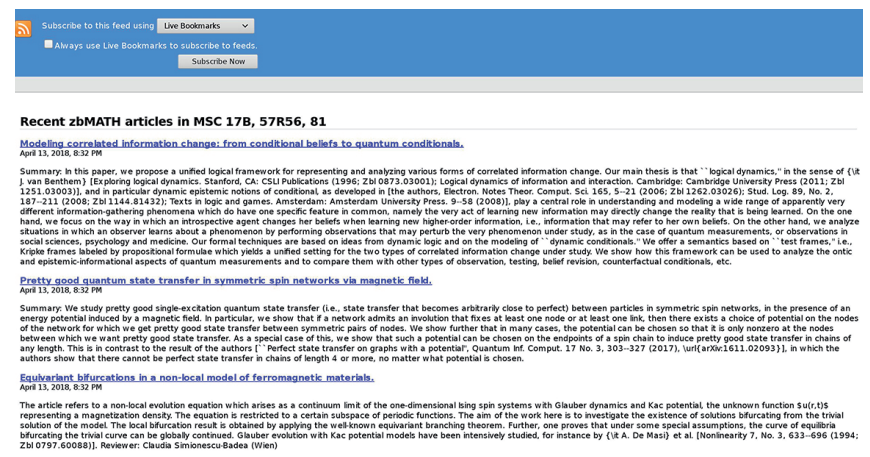




\section{Tutorial videos}

A series of videos have been produced in order to help users exploit all of zbMATH's potential. There are examples with complex queries, tricks and hints. New videos will be added to cater for newly developed features in zbMATH. You can watch these videos at the very bottom of the "About" page https://zbmath.org/about\#id_5.

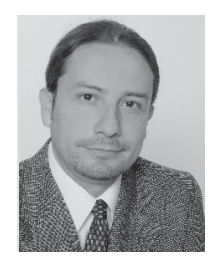

Octavio Paniagua Taboada is an editor for zbMATH at the Berlin office of FIZ Karlsruhe. He received a bachelor's degree and a Master's in mathematics from the National Autonomous University of Mexico (UNAM) and a PhD in mathematics from the Université Paris-Sud XI, Orsay, France. He has been an editor of zbMATH since January 2014 and of the EMS Newsletter since 2018.

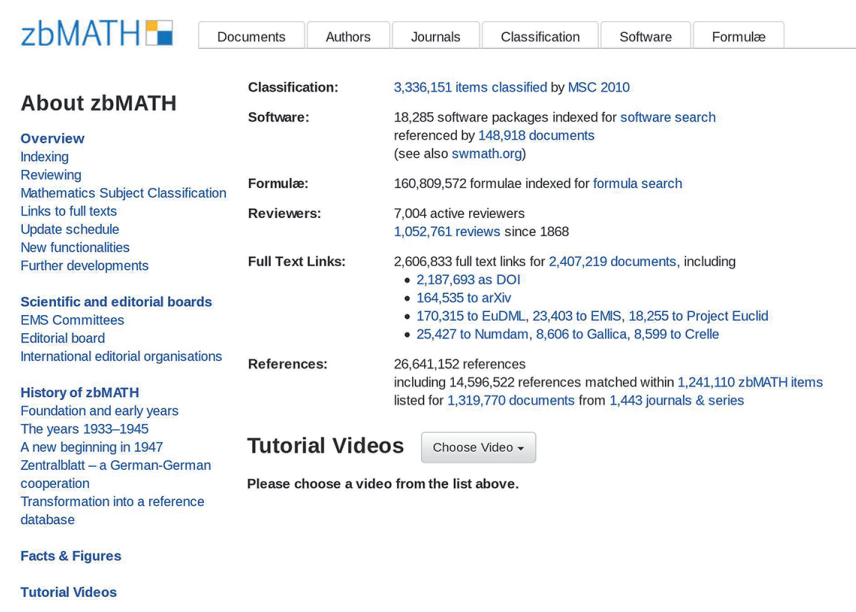

